\title{
Long-term evolution of AGB wind envelopes: Insights from hydrodynamical models
}

\author{
M. Steffen and D. Schönberner \\ Astrophysikalisches Institut Potsdam, An der Sternwarte 16, \\ 14482 Potsdam, Germany \\ R. Szczerba \\ N. Copernicus Astronomical Center, Rabianska 8, 87-100 Torun, Poland
}

\begin{abstract}
Up to now, hydrodynamical models of dust-driven AGB winds do not generally take into account the 'long-term' changes of the stellar parameters (on stellar evolution time scales of $10^{3}$ to $10^{5} \mathrm{yrs}$ ), although it is well known that the luminosity and (very likely) the mass loss rate undergo significant variations when so called 'thermal pulses' occur on the upper AGB. In this review we demonstrate that time-dependent radiation hydrodynamics calculations are needed to understand the formation, structure, and spectral energy distribution of detached dust shells detected by IRAS and ISO. Combined with appropriate models, these observations can reveal part of the previous mass loss history on the AGB and allow an empirical check of presently adopted mass loss laws.

Based on insights from hydrodynamical simulations, we discuss the two competing scenarios that have been put forward to explain the origin of the very thin molecular shells recently discovered around some carbon stars. We find that the signature of a short mass loss 'eruption' broadens quickly with time due to the related velocity gradient across the shell. Hence, this scenario is not considered a likely explanation of detached $\mathrm{CO}$ shells. On the other hand, the alternative mechanism, interaction of winds, is shown to be capable of producing very thin shells of greatly enhanced gas density in the dusty outflows from AGB stars by sweeping up matter at the interface between both type of winds.
\end{abstract}

\section{Introduction}

It is now generally accepted that the mechanism responsible for the high mass loss rates during the AGB evolution is based on the efficiency of radiation pressure on dust grains. However, it is largely unknown how the mass loss rate depends on the star's main sequence mass, its metallicity, its particular phase of evolution and pulsational properties. In order to determine the temporal behavior of the mass loss rate (exponentially increasing, interrupted, episodic ?) empirically, we have to study the radial structure of the circumstellar shells of AGB stars. In the following we briefly review different approaches to modeling circumstellar gas/dust shells and demonstrate that time-dependent model calculations including the effects of stellar evolution are needed to understand 
the formation, radial structure and spectral energy distribution of detached dust shells observed by IRAS and ISO. We present some example results obtained with a radiation hydrodynamics code recently developed as a diagnostic tool for the interpretation of IR observations of the dust emission around AGB and post-AGB stars. Comparison of such observations with appropriate models permits an empirical check of presently adopted mass loss laws, an important step towards revealing the detailed mass loss history on the AGB.

\section{Different approaches to modeling circumstellar gas/dust shells}

* Radiative transfer calculations: The consistent solution of the radiative transfer equation is much more demanding. One has to specify the density structure of the circumstellar shell (usually assuming spherical symmetry, $\left.\rho \sim r^{-\alpha}, \alpha \approx 2\right)$ and the optical dust properties. Given the parameters of the central $\operatorname{star}\left(L_{*}, T_{*}\right)$ and the inner radius of the dust shell, $r_{1}$, the requirement of dust radiative equilibrium yields the radial temperature distribution of the dust, $T_{d}(r)$, the emergent spectral energy distribution, $\lambda F_{\lambda}$, and the spatial surface brightness distribution projected onto the sky, $I_{\lambda}(p)$. This approach is widely used for the analysis of the SEDs of stars with dusty envelopes (e.g. Hashimoto 1994, 1995; Groenewegen 1995, 1997). Some authors take into account a dust size distribution (Justtanont \& Tielens 1992; Szczerba et al. 1997), and there are even codes for treating non-spherical, disk-like configurations (Men'shchikov \& Henning 1996). Lacking hydrodynamical models, some authors have tried to mimic the effect of mass loss variations by considering a sequence of density structures modified according to some adopted temporal mass loss behavior, assuming that the outflow velocity is basically independent of the mass loss rate, $v \neq f(\dot{M})$ (e.g. Bedijn 1987; Groenewegen \& de Jong 1994; Suh \& Jones 1997).

$\star \star$ Steady state hydrodynamical models: The above mentioned pure radiative transfer models ignore the fact that the problem of momentum transfer (hydrodynamics) is inherently coupled with the problem of radiative transfer: radiation pressure on dust determines the outflow velocity and hence the density structure; at the same time, the density structure determines - via radiative transfer effects - the spectrum of the photons and hence the effective radiation pressure. Over the last years, this circular problem has been addressed by Netzer \& Elitzur (1993), Habing et al. (1994), Justtanont et al. (1994), Ivezić \& Elitzur (1995), and Steffen et al. (1997a), who describe the gas/dust shell as a hydrodynamical multi-fluid system and allow for a relative motion of gas and dust. Steffen et al. (1997a) include the dynamical effects of the gas pressure which is usually ignored. With stellar parameters $\left(M_{*}, L_{*}, T_{*}\right)$, mass loss rate $(\dot{M})$ and dust properties (grain size, $a$, mass density of the grain material, $\rho_{\mathrm{I}}$, dust condensation temperature, $T_{c}$, absorption and scattering cross sections, $C_{\lambda}^{\text {abs }}, C_{\lambda}^{\text {sca }}$ and the dust-to-gas mass ratio, $\left.\delta\right)$ as input data, $r_{1}, v(r), \rho(r), v_{d}(r)$, $\rho_{d}(r), T_{d}(r)$ as well as $\lambda F_{\lambda}$ and $I_{\lambda}(p)$ are found from the solution of the coupled hydrodynamics/radiative transfer problem.

For simplicity, all these model calculations are based on the assumption of a spherically symmetric steady state flow. It has been shown that, for a given sort of grains, a sequence of steady state models with increasing mass loss rate is mapped on a simple color-color relation, with optical depth of the dust shell as 
the single independent parameter (Ivezić \& Elitzur 1995). Indeed the majority of mass losing AGB stars can be understood in terms of such steady state models characterized by a constant value of $\dot{M}$. However, even if one assumes a large variety of different grain compositions, it is impossible to explain the observed distribution of AGB objects with excess 60 and $100 \mu \mathrm{m}$ emission in the IRAS two-color-diagram (cf. Fig. 2, top).

$\star \star \star$ Time-dependent radiation hydrodynamics models: In order to properly take into account the evolutionary changes of the stellar parameters and a variable mass loss rate, it is necessary to solve the time-dependent coupled equations of hydrodynamics and radiative transfer. Given the stellar parameters $\left(M_{*}(t), L_{*}(t), T_{*}(t)\right)$, and the mass loss rate $(\dot{M}(t))$ as time-dependent external boundary values, the solution of the equations of a dust-driven stellar wind describes the structure and dynamics of the circumstellar envelope as a function of time yielding: $r_{1}(t), v(r, t), \rho(r, t), v_{d}(r, t), \rho_{d}(r, t), T_{d}(r, t)$ as well as $\lambda F_{\lambda}(t)$ and $I_{\lambda}(p, t)$. Model calculations of this type have first been performed by Vassiliadis $\&$ Wood (1992) and Szczerba \& Marten (1993). More detailed results of timedependent two-component simulations of the dusty winds of AGB stars in their final stages of evolution have recently been presented by Steffen et al. (1998). These models are further discussed in the next section.

$\star \star \star \star$ Fully self-consistent radiation hydrodynamics models: The models discussed above are far from being fully self-consistent: they cover only those parts of the circumstellar envelope that lie outside the sonic point and therefore cannot make predictions about the mass loss rate which is determined in the subsonic regions near the stellar surface. Clearly, the ultimate goal is to develop fully self-consistent physical models of circumstellar AGB shells, combining stellar pulsations, dust chemistry, hydrodynamics and stellar evolution. The most advanced models in this direction are those by Fleischer et al. (1992, $\&$ this volume; see also the review by Sedlmayr \& Dominik 1995). Similar models have been computed by Höfner \& Dorfi (1997, \& this volume). Given the stellar parameters, the surface $\mathrm{C} / \mathrm{O}$ ratio, and the pulsational properties of the star, these models give, among other things, the mass loss rate and the outflow velocity as a result. Up to now, these kinds of models are only available for carbon-rich chemistry. They are time-dependent in the sense that they include Mira-type pulsations (enforced by a piston), but they cannot yet account for the changes of the stellar parameters and surface composition on stellar evolution time scales. Clearly, a lot of work is still needed before truly self-consistent models of circumstellar AGB shells will be available.

\section{Time-dependent AGB wind models coupled to stellar evolution calculations}

We have applied time-dependent two-component hydrodynamics/radiative transfer calculations to model the dusty winds of AGB stars in their final stages of evolution. This approach allows us to take into account the evolutionary changes of the stellar parameters, including the mass loss rate, and to investigate the dynamical response of the AGB wind envelope and of the emergent spectral energy distribution to the temporal variations of $L_{*}, T_{*}$, and $\dot{M}$. The basic assumptions, numerical method and input data as well as first results have been 

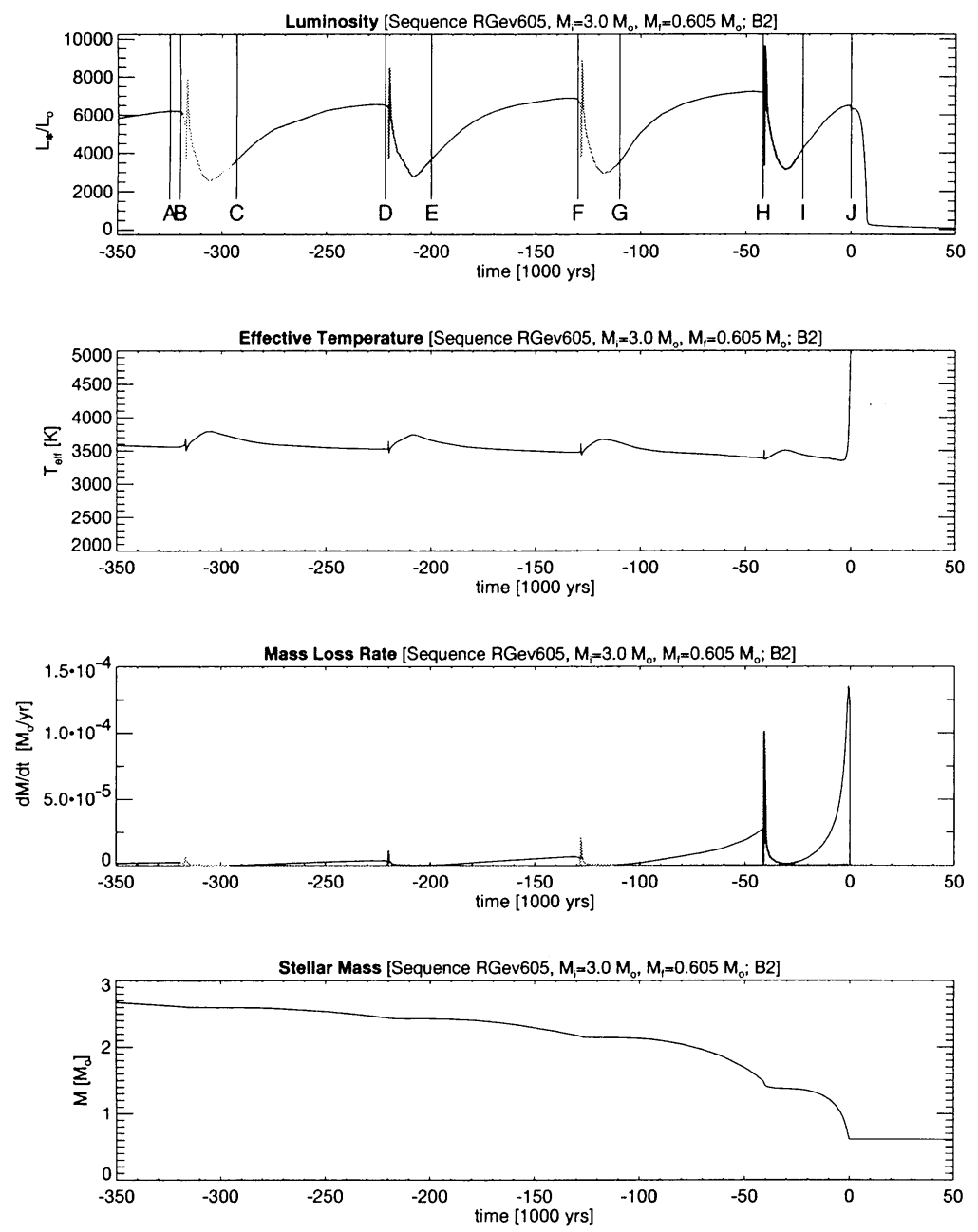

Figure 1. Luminosity, effective temperature, mass loss rate, and stellar mass versus time for the final four thermal-pulse cycles of a $3 \mathrm{M}_{\odot}$ model sequence after Blöcker (1995). Time zero marks the beginning of the model's post-AGB evolution. For further details see text.

described by Steffen et al. 1998 (see also Steffen \& Szczerba 1997; Steffen et al. 1997a,b; Schönberner et al. 1997, 1998).

The example results presented in the following are based on a stellar evolution sequence computed by Blöcker (1995) for a solar composition star of initially $3 \mathrm{M}_{\odot}$. The temporal variation of the stellar parameters and the mass loss rate over a time interval of about 350000 years, covering the final stages of evolution on the AGB, are shown in Fig. 1. The mass loss rate adopted for computing this sequence depends on the stellar parameters according to $\dot{M}=5.8510^{-14}\left(L_{*} / L_{\odot}\right)^{4.2}\left(M_{*} / M_{\odot}\right)^{-3.1}\left(T_{*}[\mathrm{~K}]\right)^{-2}$. Note that the bulk of the mass loss occurs during the final four thermal pulse cycles. These stellar evolution data are used as an inner boundary condition for the hydrodynamical sim- 
ulation of the long-term evolution of the circumstellar gas/dust shell. Mira-type stellar pulsations (with periods of a few 100 days) cannot be included explicitly (the time step of the simulations is typically $50 \mathrm{yrs}$ ) but only as an additional acceleration term representing the time-averaged pressure of shock waves.

What happens to the circumstellar envelope during a thermal pulse cycle? Before the onset of a helium shell flash (e.g.time ' $D$ ' in Fig. 1), luminosity and mass loss have been increasing slowly and steadily over several 10000 years. Hence, the velocity and density structure of the wind closely resembles that of a steady state outflow: constant velocity over a wide radial range, and $\rho \sim r^{-2}$ (except for the outermost regions beyond $r \approx 510^{17} \mathrm{~cm}$ that still exhibit the signature of the previous thermal pulse). At the same time, the emergent SED is typical for steady state conditions, characterized by a monotonically decreasing flux towards longer wavelengths. At the subsequent mass loss rate minimum (e.g. between times ' $\mathrm{D}$ ' and ' $\mathrm{E}$ ' in Fig. 1), the coupling between gas and dust has become insufficient to drive the outflow due to the very low matter densities in the inner regions. The slow $(3 \mathrm{~km} / \mathrm{s})$ gas flow is supported only by the pressure of shock waves (see above). It is only in the outer regions $\left(r>10^{17} \mathrm{~cm}\right)$ that we still see the signature of the previous phase of high mass loss rate: the gas velocity still reaches about $10 \mathrm{~km} / \mathrm{s}$, and the dust density increases distinctly, showing a local maximum near $r \approx 3 \cdot 10^{17} \mathrm{~cm}$. This detached dust shell is the result of the sharp decrease of the mass loss rate by more than one order of magnitude (from $310^{-6} \mathrm{M}_{\odot} / \mathrm{yr}$ at ' $\mathrm{D}$ ' to $110^{-7} \mathrm{M}_{\odot} / \mathrm{yr}$ about 12000 years later). In the emergent spectrum, this excess concentration of cold dust is seen as a conspicuous excess emission at 60 and $100 \mu \mathrm{m}$. In this phase, the computed radial intensity distribution (e.g. $\lambda 100 \mu \mathrm{m}$ ) shows a local maximum at distances of a few times $10^{17} \mathrm{~cm}$ from the central star, corresponding to a ring-like structure in the surface brightness. Indeed, such detached dust shells have been found by IRAS (Waters et al. 1994, Izumiura et al. 1997 [multiple shell]) and ISO (Izumiura et al. $1996 \&$ this volume). Similar results have been obtained from models with oxygen-rich circumstellar shells.

As our model runs through a thermal pulse, the emergent spectral energy distribution changes with time. This temporal variation of the SED is translated into an extended loop in the IRAS two-color-diagram. Over the time interval of 350000 yrs covered by Fig. 1, each of the four thermal pulses produces a distinct loop in the two-color-diagram (lower panel of Fig. 2). Comparison with the observed distribution of carbon stars in the IRAS two-color-diagram (top panel of Fig. 2) suggests that the objects located in region VIa are carbon stars just having suffered a thermal pulse which reduced the mass loss by at least an order of magnitude and lead to the detachment of the dust shell. Objects with steady state wind envelopes are expected to follow the simple color-color relation indicated by the dashed line (top panel of Fig. 2). Clearly, steady state models cannot explain the carbon stars observed in region VIa. For further details see Steffen et al. (1997b, 1998) and Steffen \& Szczerba (1997).

Since the location, shape and population density of the loops depend sensitively on the adopted mass loss law, the comparison of observed and computed IRAS two-color diagrams is a powerful tool to put constraints on the mass loss history on the AGB, provided that the data sets used for comparison are statistically representative. 

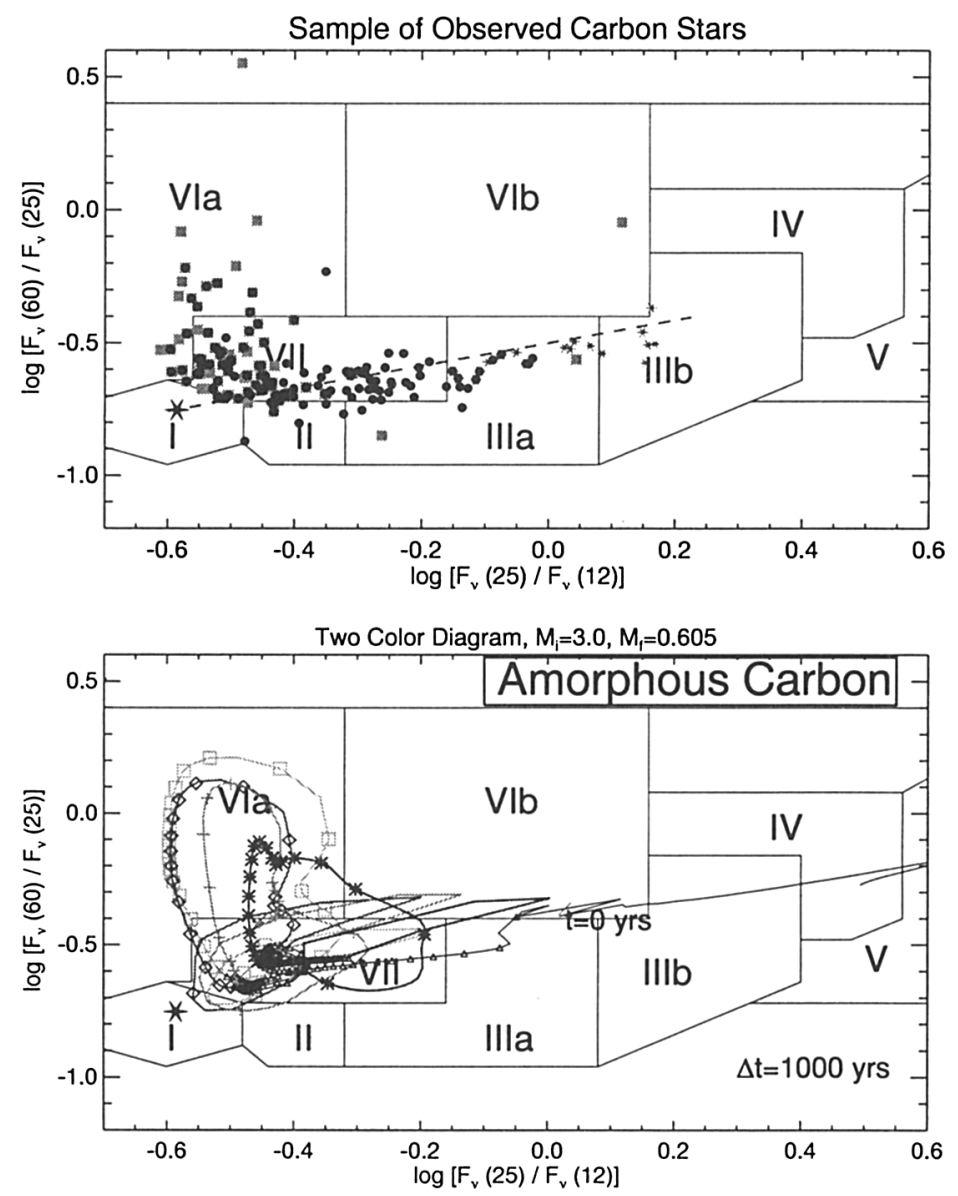

Figure 2. Top: Distribution of observed carbon stars in the IRAS [60]-[25]) vs. [25]-[12] two-color-diagram. The data for classical C-stars (circles), optical C-stars (squares) and extreme C-stars (asterisks) are courtesy of K. Volk (priv. comm.); only objects with fluxes of quality 3 at $12,25,60$, and $100 \mu \mathrm{m}$ are plotted. The big 'star' in region I is the Rayleigh Jeans point for $T=3000 \mathrm{~K}$. The dashed line delineates the color-color relation for steady state models (cf.Steffen et al. 1997a). Bottom: Computed evolution of a carbon star in the same IRAS two-color-diagram, based on the stellar evolution sequence shown in Fig. 1. The hydrodynamical simulation covers 4 thermal pulse cycles during the final 350000 years of AGB evolution, each of which produces a corresponding loop in the two-color-diagram, distinguished here by different symbols: squares (B-C), diamonds (D-E), plus-signs (F-G), and asterisks (H-I), plotted in equidistant time intervals of 1000 years, while triangles are used otherwise. The sequence starts somewhere in region VII, reaches the position marked ' $t=0$ ' at the end of the AGB and leaves the diagram to the right when entering the post-AGB phase. 


\section{On the origin of thin detached $\mathrm{CO}$ shells}

Searching for evidence of episodic mass loss of evolved stars on the AGB, Olofsson et al. (1996) noted the existence of detached narrow shells (width-to-radius $\approx 0.3 \ldots 0.4)$ in molecular line emission of $\mathrm{CO}$ around the carbon stars $\mathrm{R} \mathrm{Scl}$, U Ant, S Sct, and TT Cyg. Very recently, Olofsson et al. (1998) have succeeded in spatially resolving the CO shell around TT Cyg through interferometric observations and found an even 10 times smaller width-to-radius value of only $\approx 0.04$. Absolute radii of the CO shells are estimated to lie between about 1 and 5 times $10^{17} \mathrm{~cm}$. Since their discovery, the origin of the circumstellar $\mathrm{CO}$ shells has been a matter of debate. Two competing scenarios have been put forward to explain their existence: Mass loss 'eruption' versus two-wind interaction.

Even though no physical mechanism is presently known to be responsible for producing very short mass loss 'eruptions' (see however recent claims by Schröder et al. 1998 \& this volume), it is nevertheless tempting to relate the existence of the observed very thin $\mathrm{CO}$ shells to this presumed phenomenon. According to Olofsson et al. (1998) the CO shell of TT Cyg has a width of 2 $10^{16} \mathrm{~cm}$ and a radius of $5.110^{17} \mathrm{~cm}$. Assuming a constant expansion velocity of $13 \mathrm{~km} / \mathrm{s}$ (the measured Doppler velocity inside the CO shell), this implies a mass loss 'eruption' that happened about $12000 \mathrm{yrs}$ ago and lasted for about $500 \mathrm{yrs}$, at a rate of $\dot{M} \approx 10^{-4} \mathrm{M}_{\odot} / \mathrm{yr}$ (estimated shell mass $\approx 0.024 \mathrm{M}_{\odot}$ ).

We have constructed a schematic mass loss history in accordance with the above mentioned data for TT Cyg $\left(\dot{M}=510^{-5} \mathrm{M}_{\odot} / \mathrm{yr}\right.$ during the 'eruption'), and used it as a boundary condition for a time-dependent hydrodynamical simulation of the mass loss 'eruption' scenario. The results indicate that the outflow velocity decreases sharply from about $u_{1}=21 \mathrm{~km} / \mathrm{s}$ at the onset to $u_{2}=6 \mathrm{~km} / \mathrm{s}$ at the end of the 'eruption' due to the increasing optical depth of the ejected dust. The produced gas shell moves outward at an average speed of $\approx 15 \mathrm{~km} / \mathrm{s}$. However, the substantial velocity gradient across the shell leads to a considerable broadening of the density structure as it recedes from the star, and to a corresponding decline of its density contrast. Beyond $r=10^{17} \mathrm{~cm}$, the relative thickness of the shell remains approximately constant at a value of $\approx 0.6 \approx\left(u_{1}-u_{2}\right) /\left(u_{1}+u_{2}\right)$. This is more than one order of magnitude larger than the width observed in $\mathrm{CO}$ emission. We further point out that the amount of dust ejected by this 'eruption' is insufficient to account for the observed excess emission at $100 \mu \mathrm{m}$ (cf.e.g. Kerschbaum \& Hron 1996 for the observed SED of TT Cyg).

Interestingly enough, our simulations provide another mechanism creating thin shells of enhanced gas density: interaction of two winds of different velocity and density. While analyzing the simulations, we discovered that the wind velocity is an almost bi-modal function of the mass loss rate (cf. Fig. 3 for the behavior of mass loss rate and wind velocity during a typical thermal pulse). Briefly, once a critical mass loss rate is exceeded, the coupling between dust and gas becomes efficient and causes a sudden transition from a slower, dense, 'shock-driven' wind to a faster, less dense, 'dust-driven' wind, both type of winds carrying approximately the same mass loss rate $\left(r^{2} \rho u \approx\right.$ const.). This behavior leads to two-wind interaction: the faster wind in the inner parts of the shell runs into the slower wind in the outer parts, acting like a snow plow piling up matter into a thin shell at the interface between the different kind of winds. The 

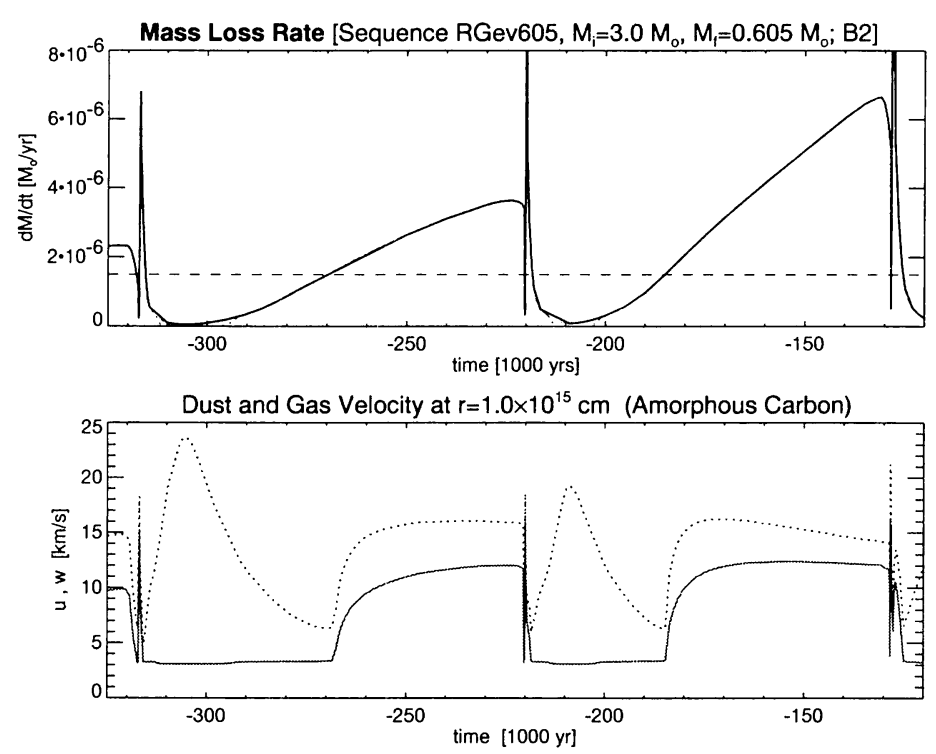

Figure 3. Top: Adopted mass loss rate over two thermal pulse cycles (Blöcker 1995). Bottom: Gas (solid) and dust (dotted) velocity in the inner wind shell $\left(r=10^{15} \mathrm{~cm}\right)$ obtained from a two-component hydrodynamical simulation as function of time, showing a characteristic bi-modal behavior of the gas velocity. When $\dot{M}>1.610^{-6} \mathrm{M}_{\odot} / \mathrm{yr}$ (dashed line in upper panel) dust/gas coupling becomes efficient and the gas outflow velocity jumps from $\approx 3$ to $\approx 12 \mathrm{~km} / \mathrm{s}$.

critical mass loss rate is $\dot{M}=1.610^{-6} \mathrm{M}_{\odot} / \mathrm{yr}$ in this example, but is expected to depend on the adopted dust properties and on the stellar parameters.

We have computed the resulting density, velocity, and temperature structure of the interaction region in a numerical experiment, using a one-component hydrodynamics code based on a high-resolution second-order Godunov-type advection scheme including a Riemann solver. This scheme adequately treats the propagation and interaction of non-linear waves in spherical geometry. Radiative cooling is treated in a simplistic way according to: $\log \left(Q_{\mathrm{rad}} / \rho\right)=4.466 *$ $(1-\exp \{-0.7177 T / 1000\})$. Starting with a steady-state model with a mass loss rate of $\dot{M}=310^{-6} \mathrm{M}_{\odot} / \mathrm{yr}$ and a constant outflow velocity of $u_{\mathrm{o}}=5 \mathrm{~km} / \mathrm{s}$, we abruptly increase the outflow velocity at the inner boundary by a factor of 3 and decrease the density of the wind by the same factor in order to keep the mass loss rate constant. We then follow the time evolution of the interaction region for about 15000 years until it has propagated outward to several $10^{17} \mathrm{~cm}$. The result is shown in Fig. 4. Indeed, a very narrow shell $\left(\Delta R_{\mathrm{s}} / R_{\mathrm{s}} \approx 0.03\right)$ of strongly (factor of 30 ) enhanced gas density has developed. Note the small velocity gradient within the compressed shell. The gas behind the inner shock is heated to about $300 \mathrm{~K}$, while the net heating behind the outer shock is negligible (a minimum temperature of $100 \mathrm{~K}$ was enforced everywhere). Probably, radiative cooling is still underestimated by our simple cooling function which we used here for illustration only. 

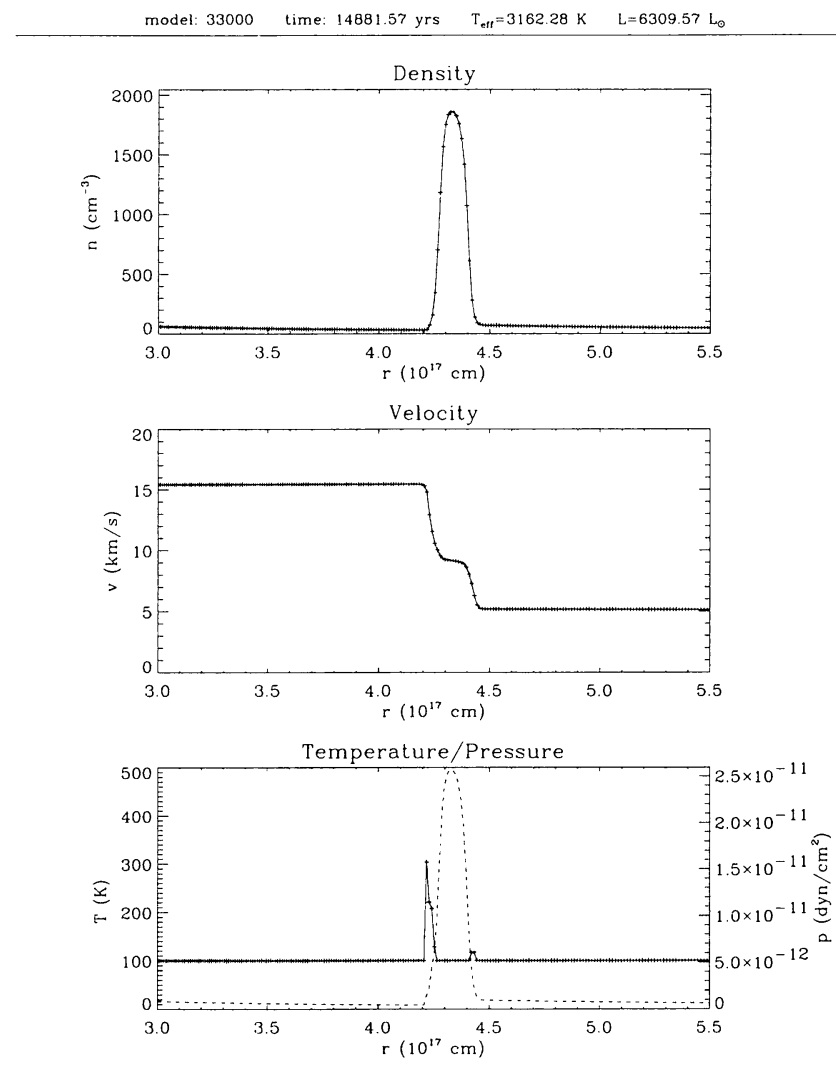

Figure 4. Particle number density (top), gas velocity (middle) and gas temperature/pressure (bottom, solid/dashed) resulting from a numerical simulation of idealized two-wind interaction. (+)-symbols indicate the numerical grid.

\section{Conclusions}

The time-dependent hydrodynamical models presented here provide a natural explanation for the existence of detached dust shells: mass loss decreases by more than one order of magnitude in the aftermath of a thermal pulse for a sufficiently long time to allow the formation of an extended, almost dust-free inner cavity. The origin of geometrically thin detached gas shells, however, is not yet sufficiently well understood. Hydrodynamical test calculations suggest that it may be difficult to produce very narrow shells by a very short mass loss 'eruption', because matter will not be ejected at a uniform velocity. On the other hand, our two-component hydrodynamical models suggest that two-wind interaction can occur in the circumstellar envelopes of AGB stars due to the almost bi-modal dependence of the outflow velocity on the mass loss rate. This behavior naturally leads to the formation of very thin shells of greatly enhanced gas density. Clearly, further research is necessary to clarify the details of this mechanism. Perhaps, the correct explanation of the remarkable $\mathrm{CO}$ shells turns out to be some combination of mass loss 'eruption' and two-wind interaction. 


\section{References}

Bedijn P.J., 1987, A\&A 186, 136

Blöcker T., 1995, A\&A 297, 727

Fleischer A.J., Gauger A., Sedlmayr E., 1992, A\&A 226, 321

Groenewegen M.A.T., 1995, A\&A 293, 463

Groenewegen M.A.T., 1997, A\&A 317, 503

Groenewegen M.A.T., de Jong T., 1994, A\&A 282, 115

Habing H.J., Tignon J., Tielens A.G.G.M., 1994, A\&A 286, 523

Hashimoto O., 1994, A\&AS 107, 445

Hashimoto O., 1995, ApJ 442, 286

Höfner S., Dorfi E., 1997, A\&A 319, 648

Ivezić Ž., Elitzur M., 1995, ApJ 445, 415

Izumiura H., Hashimoto O., Kawara K., et al., 1996, A\&A 315, L221

Izumiura H., Waters L.B.F.M., de Jong T., et al., 1997, A\&A 323, 449

Justtanont K., Tielens A.G.G.M., 1992, ApJ 389, 400

Justtanont K., Skinner C.J., Tielens A.G.G.M., 1994, ApJ 435, 852

Kerschbaum F., Hron J., 1996, A\&A 308, 489

Men'shchikov A.B., Henning T., 1996, A\&A 318, 879

Netzer N., Elitzur M., 1993, ApJ 410, 701

Olofsson H., Bergmann P., Eriksson K., Gustafsson B., 1996, A\&A 311, 587

Olofsson H., Bergmann P., Lucas R., et al., 1998, A\&A 330, L1

Schönberner D., Steffen M., Stahlberg J., Kifonidis K., Blöcker T., 1997, in Advances in Stellar Evolution, eds. R.T. Rood and A. Renzini, Cambridge University Press, p. 146

Schönberner D., Steffen M., Stahlberg J., Kifonidis K., Blöcker T., 1998, in The Carbon Star Phenomenon, Proc. IAU Symp. 177, ed. R.F. Wing, Kluwer Academic Publishers, in press

Schröder K.-P., Winters J.M., Arndt T.U., Sedlmayr E., 1998, A\&A 335, L9

Sedlmayr E., Dominik C. 1995, Ap\&SS Rev. 73, 211

Steffen M., Szczerba R., 1997, Ap\&SS 251, 131

Steffen M., Szczerba R., Men'shchikov A., Schönberner D., 1997a, A\&AS 126, 39

Steffen M., Szczerba R., Men'shchikov A., Schönberner D., 1997b, in Advances in Stellar Evolution, eds. R.T. Rood and A. Renzini, Cambridge University Press, p. 154

Steffen M., Szczerba R., Schönberner D., 1998, A\&A 337, 149

Suh K.-W., Jones T.J., 1997, ApJ 479, 918

Szczerba R., Marten H., 1993, in Mass Loss on the AGB and Beyond, ed. H.E.

Schwarz, ESO Conference and Workshop Proceedings No. 46, p. 90

Szczerba R., Omont A., Volk K., Cox P., Kwok S., 1997, A\&A 317, 859

Vassiliadis E., Wood P.R., 1992, Proc. Astron. Soc. Aust. 10 (1), 30

Waters L.B.F.M., Loup C., Kester D.J.M., et al., 1994, A\&A 281, L1 